\title{
High-Frequency, Silicon-Based Ultrasonic Nozzles Using Multiple Fourier Horns
}

\author{
Shirley C. Tsai, Yu L. Song, Terry K. Tseng, Yuan F. Chou, Wei J. Chen, \\ and Chen S. Tsai, Fellow, IEEE
}

\begin{abstract}
This paper presents the design, simulation, and characterization of microfabricated $0.5 \mathrm{MHz}$, siliconbased, ultrasonic nozzles. Each nozzle is made of a piezoelectric drive section and a silicon resonator consisting of multiple Fourier horns, each with half wavelength design and twice amplitude magnification. Results of finite element three-dimensional (3-D) simulation using a commercial program predicted existence of one resonant frequency of pure longitudinal vibration. Both impedance analysis and measurement of longitudinal vibration confirmed the simulation results with one pure longitudinal vibration mode at the resonant frequency in excellent agreement with the design value. Furthermore, at the resonant frequency, the measured longitudinal vibration amplitude at the nozzle tip increases as the number of Fourier horns (n) increases in good agreement with the theoretical values of $2^{n}$. Using this design, very high vibration amplitude gain at the nozzle tip can be achieved with no reduction in the tip cross-sectional area for contact of liquid to be atomized. Therefore, the required electric drive power should be drastically reduced, decreasing the likelihood of transducer failure in ultrasonic atomization.
\end{abstract}

\section{INTRODUCTION}

Silicon-BASED ultrasonic nozzles have a number of advantages over conventional, metal-based, bulk-type ultrasonic nozzles [1]. Such a silicon-based ultrasonic nozzle at $72 \mathrm{kHz}$ was constructed previously [1]. Silicon possesses a relatively large electromechanical coupling coefficient, a high-acoustic velocity, and a high potential for mass production of any resonator profile by micro electro mechanical system (MEMS)-based fabrication technology. These advantages enable silicon-based ultrasonic nozzles to overcome the $120-\mathrm{kHz}$ frequency limitation of conventional, metal-based, bulk-type ultrasonic nozzles [2]. Together with ultrasound-modulated, twin-fluid (UMTF) atomization (spray) technique [3], [4] that uses air to assist ultrasonic atomization, further advantages can be realized. Specifically, UMTF atomization has been demonstrated to

Manuscript received August 7, 2003; accepted October 13, 2003. Support by the National Science Foundation, USA, under Grant \#CTS-98120050, the National Science Council and the Academia Sinica, Taiwan is gratefully acknowledged.

S. C. Tsai, Y. L. Song, T. K. Tseng, W. J. Chen, and C. S. Tsai are with the Institute for Applied Science and Engineering Research, Academia Sinica, Taipei 115, Taiwan (sctsai@phys.sinica.edu.tw).

S. C. Tsai is also with the Dept. of Chemical Engineering, California State Univ., Long Beach, CA 90840, USA.

Y. F. Chou is with the Dept. of Mechanical Engineering, National Taiwan University, Taipei, Taiwan.

C. S. Tsai is also with the Dept. of Electrical Eng. and Computer Science, Univ. of California, Irvine, CA 92697, USA. produce much smaller and more uniform drops than the conventional ultrasonic atomization at the same fundamental frequency [3]-[5]. In fact, the peak drop diameter (the diameter in which the peak of a drop-size distribution occurs) obtained by UMTF atomization was found equal to the wavelength of the capillary waves generated by the third harmonic frequency [3]-[5]. In other words, an UMTF atomizer operating at $0.8 \mathrm{MHz}$ fundamental should produce uniform drops the same size as those produced by a conventional, ultrasonic nozzle operating at $2.5 \mathrm{MHz}$.

Ultrasonic nebulizers are commercially available at frequencies as high as $2.5 \mathrm{MHz}$; however, they require very high electric drive power, which severely limits atomization rates in order to avoid transducer failure. Moreover, because the transducer must be in direct contact with the precursor solution, performance degrades quickly over time. In contrast, an UMTF atomizer is made of an annulus for airflow and an ultrasonic nozzle with a central channel for liquid flow. Its piezoelectric transducers are not in direct contact with the precursor liquids and, thus, avoid interference with transducer operation as in the case of conventional ultrasonic atomization using a nebulizer. In addition, an UMTF atomizer consisting of a $0.8 \mathrm{MHz}$ ultrasonic nozzle will generate the same drop sizes $(5-8 \mu \mathrm{m}$ in diameter) as a $2.5 \mathrm{MHz}$ ultrasonic nebulizer as mentioned previously. Arrays of such high-frequency silicon ultrasonic nozzles can be fabricated using the MEMS-based technology and used for mass production of nanoparticles of advanced functional materials by spray pyrolysis. In fact, uniform spherical particles of yttria stabilized zirconia $68 \mathrm{~nm}$ in diameter have been produced by spray pyrolysis of 5 $8 \mu \mathrm{m}$ precursor drops, generated by an ultrasonic nebulizer at $2.5 \mathrm{MHz}[6]$.

This paper reports on the design, simulation, measurement, and characterization of $0.5 \mathrm{MHz}$ ultrasonic nozzles fabricated using MEMS technology in (001) silicon wafers. To the best of our knowledge, no ultrasonic nozzle at such a high frequency has ever been reported. As shown in Fig. 1, the nozzle is composed of a transducer drive section and a silicon resonator with a central channel for liquid flow. The drive section consists of a pair of piezoelectric transducer (PZT) plates with a rectangular section of silicon bonded in between. The silicon resonator is made of one or multiple Fourier horns in cascade, each with half-wavelength design and vibration amplitude magnification of two. The nozzle is geometrically configured such that excitation of the PZT plates creates a standing wave through the nozzle with maximum longitudinal vibration at the nozzle tip. 


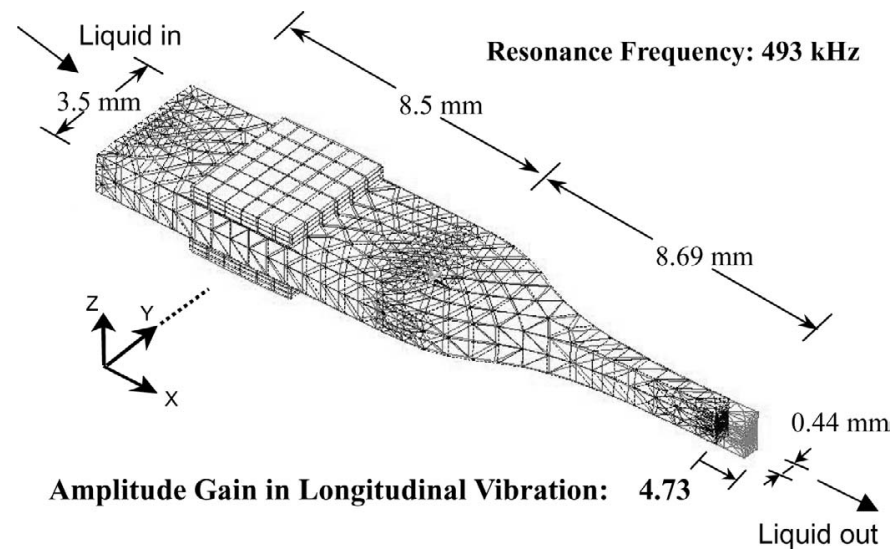

Fig. 1. A silicon-based, Fourier-horn, ultrasonic nozzle with $0.5 \mathrm{MHz}$ ultrasonic frequency and magnification of five.

As the liquid issues from the nozzle tip, a capillary wave is generated on the liquid surface [7] with initial amplitude proportional to the amplitude of the longitudinal vibration of the nozzle tip. Traveling in the direction of liquid flow (X-axis), this temporally unstable liquid capillary wave eventually collapses into drops with a diameter inversely proportional to the ultrasonic frequency [5], [8]. A unique advantage of the multiple-horn nozzle is that the longitudinal vibration amplitude gain at the nozzle tip can be increased considerably without a reduction in tip crosssectional area. This cannot be achieved with a single-horn nozzle.

\section{Theoretical Basis for Design of Fourier HORN}

The novel feature of the high-frequency (megahertz) ultrasonic nozzle presented here is the cascade design of multiple Fourier horns that vibrate longitudinally at the same resonant frequency as a single Fourier horn, and the vibration amplitude at the nozzle tip is greatly amplified. Fig. 1 shows the rectangular global coordinate system for design and simulation of the Fourier horn profile. The X-axis, parallel to the central channel for liquid flow, is in the direction of $\langle 110\rangle$, the primary flat on the silicon wafer. The Z-axis, perpendicular to the wafer plane, is the direction of polarization of the PZT (or the direction of the electric field). The governing 1-D equations for simulation of each Fourier horn are written with abbreviated notation in which $1,2,3,4,5$, and 6 refer to $\mathrm{xx}, \mathrm{yy}, \mathrm{zz}, \mathrm{yz}, \mathrm{xz}$, and $\mathrm{xy}$, respectively.

The 1-D model for longitudinal particle displacement (or vibration amplitude) $u_{x}$ of the horn with varying crosssectional area $A(x)$ at coordinate $x$ is:

$$
A(x) \frac{\partial^{2}}{\partial t^{2}} u_{x}=V_{a}^{2} \frac{\partial}{\partial x}\left(A(x) \frac{\partial}{\partial x} u_{x}\right),
$$

where the acoustic velocity $V_{a}$ is equal to $\sqrt{Y / \rho}$, in which $Y$ and $\rho$ are the Young's modulus and the density of sili- con, respectively. Eq. (1) can be rewritten in the following nondimensional form:

$$
U^{\prime \prime}(X)+U^{\prime}(X) \frac{d}{d X}(\ln A(X))+\Omega^{2} U(X)=0,
$$

where $U=u_{x} / l, X=x / l, \Omega=2 \pi l / \lambda . l$ and $\lambda$ are the horn length and the acoustic wavelength, respectively. From (2), the following basic equation for design is obtained:

$$
\frac{d}{d X}(\ln A(X))=-\frac{U^{\prime \prime}(X)+\Omega^{2} U(X)}{U^{\prime}(X)} .
$$

The boundary conditions for the Fourier horn [9] are:

- The two ends of the horn are stress free, namely, $U^{\prime}(0)=0$ and $U^{\prime}(1)=0$.

- The ratio of the amplitude of vibration at the two ends is $-\mathrm{M}$, namely, $U(0)=U_{0}$ and $U(1)=-\mathrm{M} U_{0}$.

- The slope of the lateral surface is finite and continuous everywhere, and $U^{\prime}(X)$ has no zeros in the range $0<$ $X<1$, except the essential first-order zeros at $X=0$ and $X=1$. This condition combined with (2) gives rise to two necessary conditions: $U^{\prime \prime}(0)=-\Omega^{2} U_{0}$, and $U^{\prime \prime}(1)=-\mathrm{M} \Omega^{2} U_{0}$.

- The surface slope at the low amplitude end is set to be zero, namely, $\frac{d}{d X}(\ln A) \rightarrow 0$ as $X \rightarrow 0$. Thus, $U^{\prime \prime \prime}(0)=$ 0 ; and $U^{\prime \prime \prime}(1)$ is also set to zero for symmetry.

The above boundary conditions are used to determine the coefficients $\alpha_{k}$ 's of the normalized particle displacement $U(X)$ that is expressed as a series:

$$
U(X)=\sum_{k=0}^{4} \alpha_{k} \cos (k \pi X) .
$$

Once $U(X)$ and its derivatives are determined, the cross-sectional area $A(X)$ can be obtained by integration of (3). Because the thickness of the silicon wafer is fixed, the profile of the horn is thus obtained. Note that the coefficient $\alpha_{4}$ of $\cos (4 \pi X)$ is the only free parameter in the fourth order Fourier horn once $M$ and $\Omega$ (or length $l$ of the vibrator) are specified. With the definitions of $\alpha=[32 /(\mathrm{M}-1)]\left(\alpha_{4} / U_{0}\right)$ and $\gamma=\Omega / \pi$ (the horn length normalized to half wavelength), the range of the normalized horn length $\gamma$ for a given $\alpha$ in which the maximum and minimum cross-sectional areas occur at the ends of the Fourier horn is shown in Fig. 2 for the case with $\mathrm{M}=2$. Note that only in the region encircled by $\mathrm{ABCD}$ of the figure, the aforementioned condition that $U^{\prime}(X)$ has no zeros in the range $0<X<1$ required of a Fourier horn is satisfied. The flexural compliance increases with increasing ratio (Ra) of the maximum-to-minimum cross-sectional areas of the horn, and the maximum cross-sectional dimension must be small compared to the wavelength, say, less than $\lambda / 4$ [9]. Therefore, the length of the Fourier horn should be on the order of $\lambda / 2$ based on Fig. 2. A normalized horn length $\gamma$ of 1.10 and the corresponding $\alpha$ of -0.19 that gave rise to the smallest Ra (see Fig. 2) were found to give the best mode shape by simulation for the $0.5 \mathrm{MHz}$ Fourier horn reported in this article. 


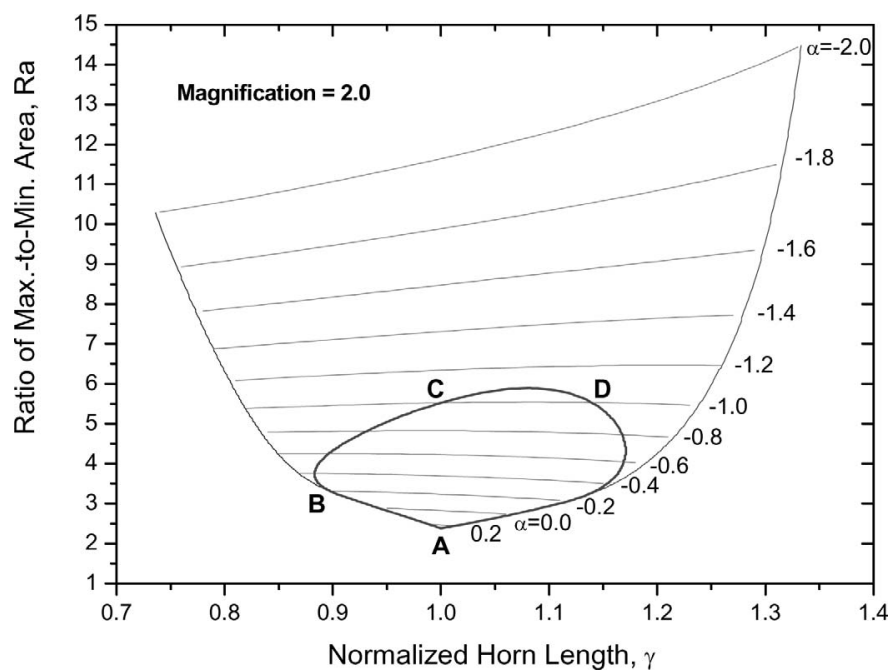

Fig. 2. The ratio of maximum to minimum cross-sectional areas as functions of normalized horn length for magnification of 2 .

\section{Constitutive Equations}

For simplicity, the constitutive equations of the 1-D model used in the simulation are given here for the case of longitudinal vibration along the $\mathrm{X}$-axis. For nonpiezoelectric materials, such as silicon, the constitutive equations consist of the strain $S_{1}-$ stress $T_{1}$ terms only. For piezoelectric materials such as PZT-5H used in the transducer drive section, the constitutive equations consist of the electrical displacement $D_{3}$ - electric field $E_{3}$ terms in addition to the $S_{1}-T_{1}$ terms. They are given as follows:

$$
\begin{aligned}
S_{1} & =s_{11}^{E} T_{1}+d_{13} E_{3}, \\
D_{3} & =\varepsilon_{33}^{T} E_{3}+d_{13} T_{1} .
\end{aligned}
$$

Rearrangements of these equations yield:

$$
\begin{aligned}
T_{1} & =\frac{1}{s_{11}^{E}}-\frac{d_{13}}{s_{11}^{E}} E_{3}, \\
D_{3} & =\left(\varepsilon_{33}^{T}-\frac{d_{13}^{2}}{s_{11}^{E} \varepsilon_{33}^{T}}\right) E_{3}+\frac{d_{13}}{s_{11}^{E}} S_{1},
\end{aligned}
$$

where the strain $S_{1}=\frac{\partial u_{x}}{\partial x}, s_{11}$ and $d_{13}$ are components of elastic compliance matrix $[s]$ and piezoelectric strain matrix $[d]$, respectively. The elastic compliance matrix is the inverse of the elastic stiffness matrix $[c][10],[11]$, namely, $[s]=[c]^{-1}$. The Young's modulus $Y_{\mathrm{mm}}$, shear modulus $G_{\mathrm{mn}}$, and Poisson ratio $\nu_{\mathrm{mn}}$ are equal to $s_{\mathrm{mm}}^{-1}$, $s_{\mathrm{mn}}^{-1}$, and $-s_{\mathrm{nm}} / s_{\mathrm{mm}}$, respectively [10]. Length-clamped (i.e., $\left.S_{1}=0\right)$ permittivity $\varepsilon_{33}^{\mathrm{LS}}$, and constant-stress permittivity $\varepsilon_{33}^{T}$ are related by $\varepsilon_{33}^{L S}=\varepsilon_{33}^{T}-\frac{d_{13}}{S_{11}^{k}}$. The electrical condition is $\frac{\partial E_{3}}{\partial x}=0$ for electroded thin plates, and the mechanical boundary condition for the transducer plate is also stress-free at both ends, namely, $T_{1}=0$ at $x= \pm w / 2$, where $w$ is the length of the transducer plate. In other words, the strain at the two ends is equal to $d_{13} \mathrm{~V} / b$, where $\mathrm{V}$ is the applied voltage and $b$ is the thickness of the transducer plate.
TABLE I

Material Properties of Silicon (Si).

\begin{tabular}{cccc}
\hline & $\mathrm{X}$ & $\mathrm{Y}$ & $\mathrm{Z}$ \\
& $\langle 110\rangle$ & $\langle\overline{1} 10\rangle$ & $\langle 001\rangle$ \\
\hline Density $\left(\mathrm{kg} / \mathrm{m}^{3}\right)$ & & 2329 & \\
\hline Young's modulus $Y$ & $Y_{11}$ & $Y_{22}$ & $Y_{33}$ \\
$\left(10^{10} \mathrm{~N} / \mathrm{m}^{2}\right)$ & 16.91 & 16.91 & 13.013 \\
Poisson's ratio & $\nu_{12}$ & $\nu_{23}$ & $\nu_{31}$ \\
$\nu$ & 0.0622 & 0.3617 & 0.2783 \\
Shear modulus $G$ & $G_{12}$ & $G_{23}$ & $G_{31}$ \\
$\left(10^{10} \mathrm{~N} / \mathrm{m}^{2}\right)$ & 5.09 & 7.96 & 7.96 \\
\hline
\end{tabular}

TABLE II

Material Properties of Piezoelectric Transducer PZT-5H.

\begin{tabular}{cccc}
\hline & $\mathrm{X}-$ & $\mathrm{Y}-$ & $\mathrm{Z}-$ \\
\hline Density $\left(\mathrm{kg} / \mathrm{m}^{3}\right)$ & & 7500 & \\
\hline Permittivity $\varepsilon$ & & & \\
$\left(10^{-8} \mathrm{C}^{2} \mathrm{~J}^{-1} \mathrm{~m}^{-1}\right)$ & 1.505 & 1.505 & 1.302 \\
& $c_{11}$ & $c_{12}$ & $c_{13}$ \\
& 12.6 & 7.95 & 8.41 \\
{$\left[c_{\mathrm{MN}}\right]$ Elastic stiffness } & $c_{22}$ & $c_{23}$ & $c_{33}$ \\
constant & 12.6 & 8.41 & 11.7 \\
$\left(10^{10} \mathrm{~N} / \mathrm{m}^{2}\right)$ & $c_{44}$ & $c_{55}$ & $c_{66}$ \\
& 2.3 & 2.3 & 2.35 \\
& $d_{13}$ & $d_{23}$ & $d_{33}$ \\
{$\left[d_{\mathrm{Mn}}\right]$ Piezoelectric strain } & -2.74 & -2.74 & 5.93 \\
constant & $d_{42}$ & $d_{51}$ & \\
$\left(10^{-10} \mathrm{C} / \mathrm{N}\right)$ & 7.41 & 7.41 & \\
Young's modulus $Y$ & $Y_{11}$ & $Y_{22}$ & $Y_{33}$ \\
$\left(10^{10} \mathrm{~N} / \mathrm{m}^{2}\right)$ & 5.988 & 5.988 & 4.808 \\
Poisson's ratio & $\nu_{12}$ & $\nu_{23}$ & $\nu_{31}$ \\
$\nu$ & 0.287 & 0.509 & 0.409 \\
Shear modulus $G$ & $G_{12}$ & $G_{23}$ & $G_{31}$ \\
$\left(10^{10} \mathrm{~N} / \mathrm{m}^{2}\right)$ & 2.347 & 2.299 & 2.299 \\
\hline
\end{tabular}

The material properties of silicon and PZT-5H used in simulation are given in Tables I and II [10], [11], respectively. Note that in Table II subscripts $\mathrm{M}$ and $\mathrm{N}$ in the elastic stiffness matrix $\left[c_{\mathrm{MN}}\right]$ and the piezoelectric strain constants $\left[d_{\mathrm{Mn}}\right]$ refer to 1 to 6 in the abbreviation notation. The Young's modulus, shear modulus, and Poisson's ratio calculated from the elastic stiffness constants for PZT-5H also are listed in Table II for comparison.

\section{Simulation Procedures AND Characterization Methods}

The 3-D simulation was first carried out using the finiteelement method (FEM) with the commercial ANSYS (ANSYS Inc., Canonsburg, PA) program for the resonator horn block to determine the resonant frequency with maximum longitudinal vibration at the horn tip. Subsequently, the lengths of the silicon and the PZT plates in the transducer drive section were adjusted to obtain a resonant frequency that matched the value of the horn block. The transducer drive block and the resonator block, each with a central 
channel, then were glued together to form the nozzle. Simulation subsequently was carried out for the nozzle to determine its resonant frequencies. Note that the very thin bonding layers that glued the PZT plates to the silicon blocks were found to have no significant effects on the simulation results reported here. A brief description of the major simulation steps now follows.

A 1-D profile of the Fourier horn resonator was first obtained by integration of (3) using a FORTRAN program based on Gauss-Kronrod rules [12], [13]. From the 1-D Fourier horn profile, a 3-D resonator block was constructed using the ANSYS program. In the simulation, element solid 64 and coupled field/scalar brick 5 were selected for the silicon and PZT plates, respectively. Provided with an adequate number of modes and the materials properties given in Tables I and II, the "modal" type of analysis in the ANSYS program with "block lanczos" mode extraction method was able to identify a series of resonant frequencies and the corresponding vibration mode shapes for the nozzle [13].

The aforementioned Fourier horn profile also was used in the layout of the mask for fabrication of 1-, 3-, and 5 -Fourier horn nozzles. Major fabrication steps for the silicon resonator halves (including the base sections where PZT plates are to be bonded) using MEMS techniques are illustrated in Fig. 3. For simplicity, the horn profile is not shown in Fig. 3. Inductive coupled plasma (ICP) etching, instead of wet etching, was used because of its capability to produce a smooth and precision finish in cutting through the $530-\mu \mathrm{m}$ thick wafer to make resonator halves with a rectangular trough $70 \mu \mathrm{m}$ deep and $200 \mu \mathrm{m}$ wide. Subsequently, two resonator halves were glued together to form a central rectangular channel $(140 \mu \mathrm{m} \times 200 \mu \mathrm{m})$ for liquid flow. Two PZT plates, one on each side, then were bonded to the resonator at the base section using silver paste; the central line of the PZT plates was aligned with the nodes of the resonator base section. The PZT plates were connected electrically to a radio frequency signal generator using coated copper wires $50 \mu \mathrm{m}$ in diameter. An illustration of such micro fabricated, 5 -horn nozzle with $0.5 \mathrm{MHz}$ design frequency is shown in Fig. 4. The impedance of the microfabricated nozzles was measured using an Agilent Impedance Analyzer Model 4294A (Agilent, Palo Alto, CA).

A schematic diagram of the setup for measuring the longitudinal vibration at the nozzle tip is shown in Fig. 5. The pair of PZTs of the silicon-based, ultrasonic nozzle is driven by the alternating current (AC) electrical signal from an Agilent Function Generator Model 33120A after amplification by Amplifier Research Model 75A250 (Amplifier Research, Souderton, PA). An Agilent 2-channel Oscilloscope 54621A monitors the AC drive signal and provides an external triggering of the Polytec Ultrasonics Vibrometer Controller Model OFV2700 (Polytech GmbH, Waldbronn, Germany). The controller is part of the Polytec laser Doppler vibrometer (LDV) that also contains a Polytec Fiber Interferometer Model OFV 511. A He-Ne laser at $632.8 \mathrm{~nm}$ wavelength in the interferometer is di-
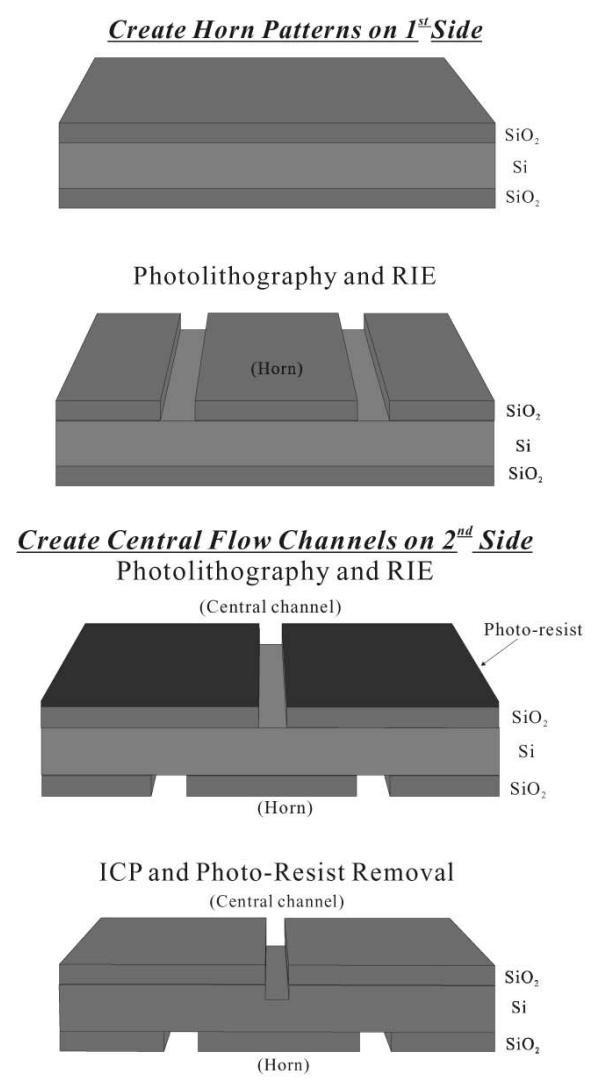

Coating of Al Film, Photolithography and Al Etching on $1^{\text {st }} \underline{\text { Side }}$

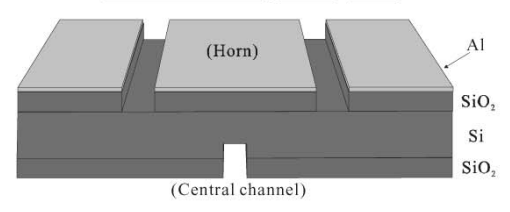

Wafer Bonding and ICP Etching

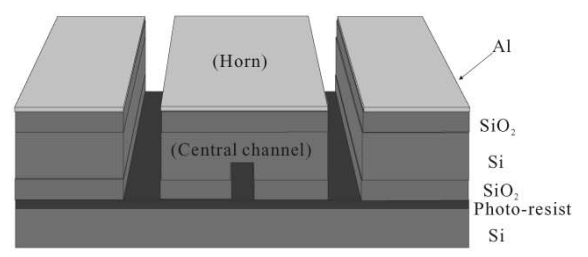

Al Stripping and Horn Release

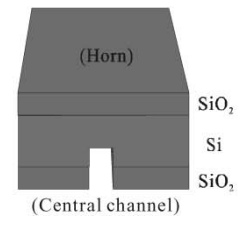

Fig. 3. Microfabrication steps for manufacture of the silicon-based, ultrasonic nozzles. 


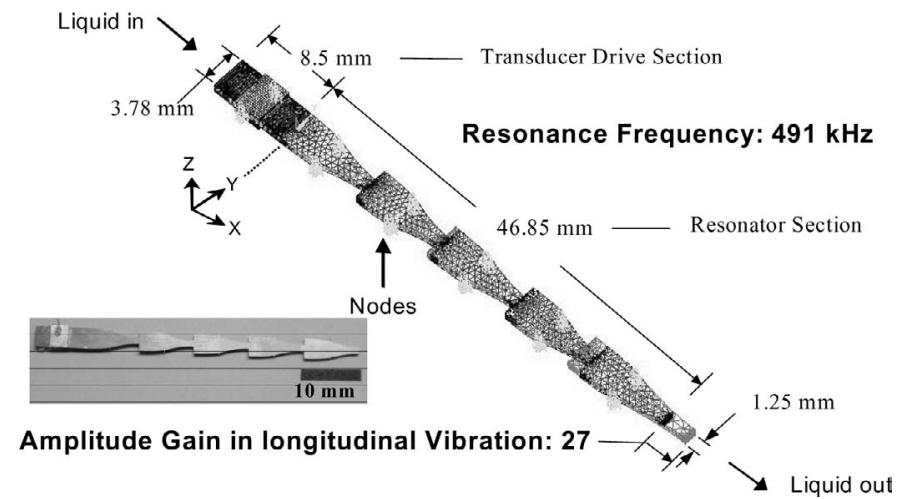

Fig. 4. Simulation results of a 5-Fourier horn, ultrasonic nozzle with $0.5 \mathrm{MHz}$ design frequency.

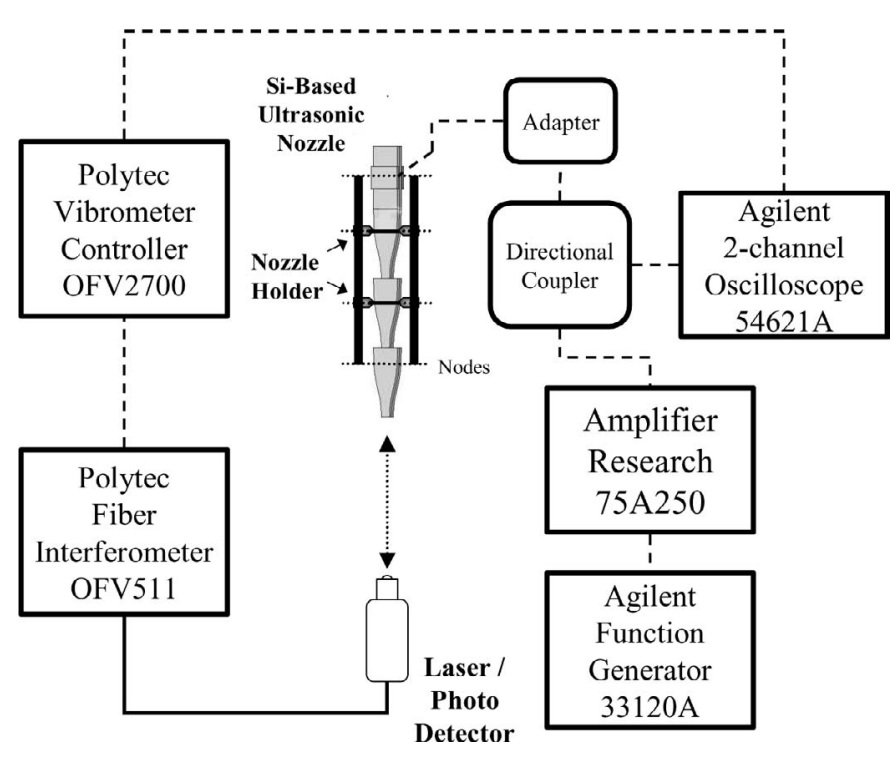

Fig. 5. Schematic diagram for measurement of longitudinal vibration at the nozzle tip.

vided into a reference beam and a probe beam. The probe beam traveling in a single fiber optic cable is focused and directed to the vibrating nozzle tip surface at normal incidence. The photo detector measures the time dependent intensity of the mixed light of the probe and the reference beams. The resulting (beat) frequency of the mixed light is just the Doppler frequency shift that is proportional to the tip vibration velocity along the axis of the probe beam. The Polytec Ultrasonics Vibrometer Controller converts the resulting frequency response into time-dependent voltage output with a conversion factor of $125 \mathrm{~mm} / \mathrm{s} / \mathrm{V}$. The maximum output voltage of the instrument is $30 \mathrm{~V}$ peakto-peak corresponding to a peak-to-peak displacement of $7.5 \mu \mathrm{m}$ at $0.5 \mathrm{MHz}$.

Longitudinal vibration at the nozzle base also was measured to provide a reference for experimental determination of the vibration amplitude magnification (or gain) at the nozzle tip.
TABLE III

Comparison of Resonant Frequencies of the Microfabricated, 5-Horn Nozzle Measured by Impedance Analysis with Simulation Results.

\begin{tabular}{|c|c|c|c|c|c|c|}
\hline & \multicolumn{3}{|c|}{ Measured groups } & \multicolumn{3}{|c|}{$\begin{array}{l}\text { Resonant frequency } \\
\text { from simulation }(\mathrm{kHz})\end{array}$} \\
\hline & 5 -horn & & -horn & & & \\
\hline 1 & $328 \mathrm{kHz}$ & 1 & $334 \mathrm{kHz}$ & $\underline{310}$ & $\underline{330}$ & \\
\hline 2 & $496 \mathrm{kHz}$ & 2 & $496 \mathrm{kHz}$ & 479 & $\underline{491}$ & 497 \\
\hline 3 & $534 \mathrm{kHz}$ & 3 & $530 \mathrm{kHz}$ & 514 & 539 & \\
\hline 4 & $582 \mathrm{kHz}$ & 4 & $604 \mathrm{kHz}$ & 588 & & \\
\hline
\end{tabular}

\section{REsults And Discussion}

\section{A. Simulation Results}

As shown in Fig. 4, simulation for a 5-Fourier horn nozzle with a design frequency of $0.5 \mathrm{MHz}$ and vibration amplitude magnification of two for each horn produced a pure longitudinal vibration at a resonant frequency of $491 \mathrm{kHz}$. Animation using the ANSYS program showed that, at this frequency, the horns in the cascade vibrate as individual units with no flexural or lateral motion. Furthermore, the maximum magnitude of the longitudinal vibration at the horn tip increased progressively in the forward direction. An amplitude gain of 27 (theoretical design $2^{5}=32$ ) was obtained as shown in Fig. 4 at the tip of the 5-horn nozzle.

It should be stressed that the 5-horn nozzle achieved such a high amplitude gain without reduction in the tip width. A comparison of Fig. 4 to Fig. 1 shows that the tip of the 5-horn nozzle is actually much wider (1.25 versus $0.44 \mathrm{~mm}$ ) than that of the single horn nozzle with a much smaller gain (4.7 in Fig. 1 versus 27 in Fig. 4). The larger tip size facilitates fabrication of the nozzle with a $140 \mu \mathrm{m} \times 200 \mu \mathrm{m}$ central channel for liquid flow. It also provides a larger contact area in which the issuing liquid experiences the maximum longitudinal vibration and, thus, sets up capillary waves on the liquid surface, which eventually collapse into drops or sprays [5], [8].

The resonant frequency of $491 \mathrm{kHz}$ with the desired longitudinal vibration mode varied only by $\pm 1 \mathrm{kHz}$ as the number of Fourier horns in the cascade (n) decreased from five to one. At this resonant frequency, the vibration amplitude magnification (or gain, equal to the longitudinal vibration at the tip divided by that at the base) of the n-horn nozzle increased proportionally to $2^{\mathrm{n}}$.

Two more pure longitudinal modes at the resonant frequencies of 310 and $330 \mathrm{kHz}$ also were obtained in the ANSYS simulation; but the longitudinal vibration resulted from four horns as a vibrating unit. The simulation also yielded many theoretically possible flexural modes, but not all were experimentally detected by impedance analysis. Therefore, only the resonant frequencies identified by the impedance analysis are listed in Table III with the longitudinal modes underlined. 
(a)

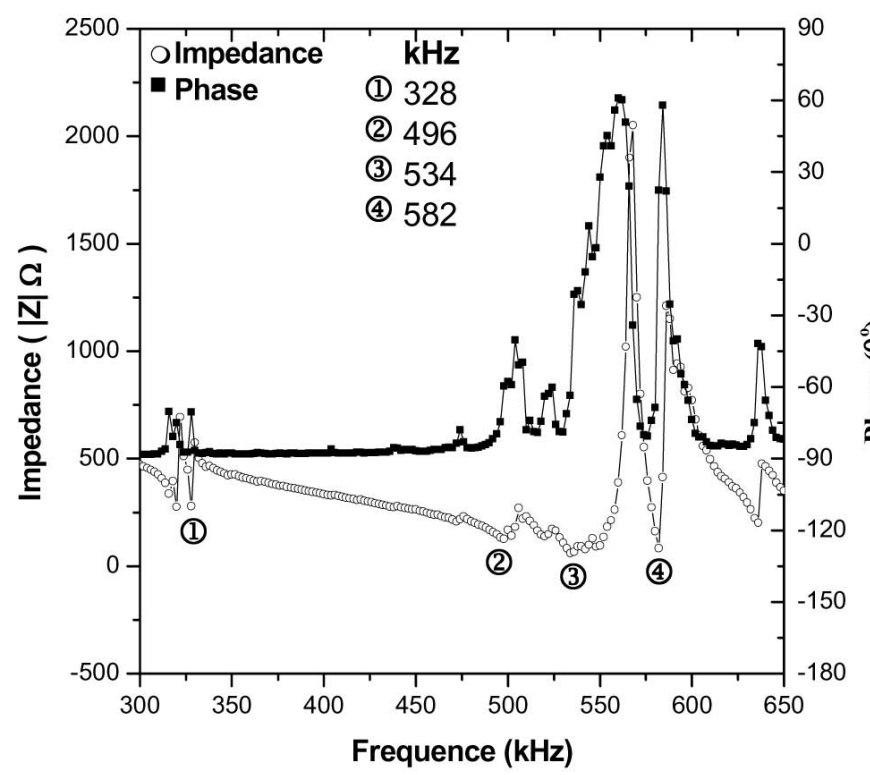

(b)

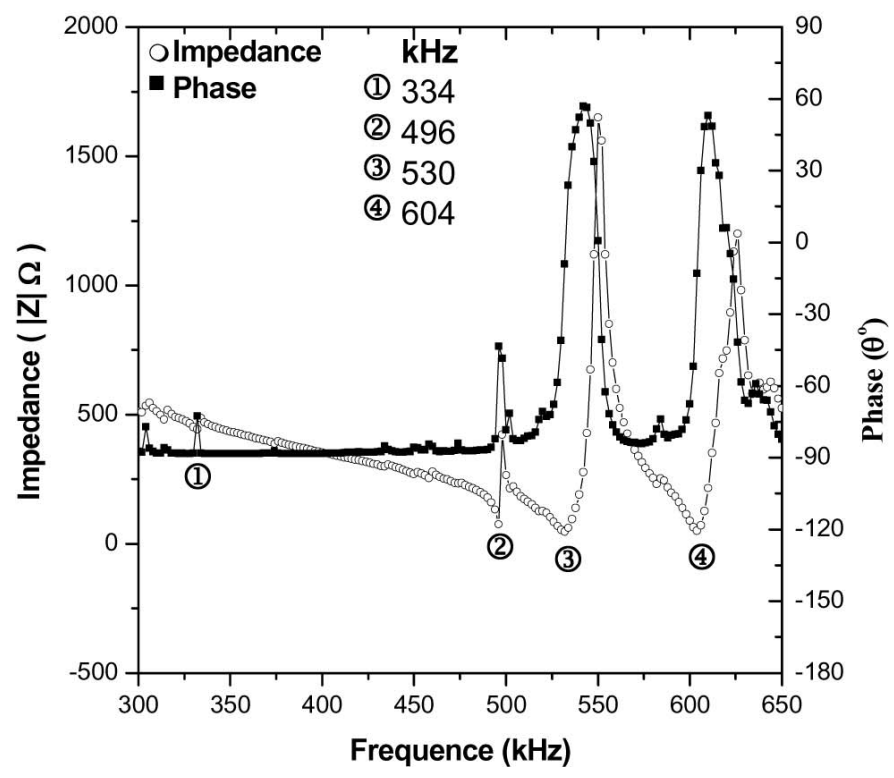

Fig. 6. Measured plots of electrical impedance and phase angle versus frequency for silicon-based, ultrasonic nozzles with design frequency of $0.5 \mathrm{MHz}$ for (a) 5-horn and (b) 1-horn.

\section{B. Experimental Verifications}

The theoretically predicted pure longitudinal vibration at $491 \pm 1 \mathrm{kHz}$ is clearly present, namely, group 2 in the frequency spectra measured by impedance analysis summarized in Table III. Its frequency is slightly shifted to $496 \mathrm{kHz}$ for both the 5-horn and the 1-horn nozzles based on the frequency spectra as shown in Figs. 6(a) and (b), respectively. Note that all resonant frequencies of the frequency spectra in these figures are divided into four groups in Table III. The other pure longitudinal modes are included in groups 1 at $328 \mathrm{kHz}$ and $334 \mathrm{kHz}$ for the 5-horn

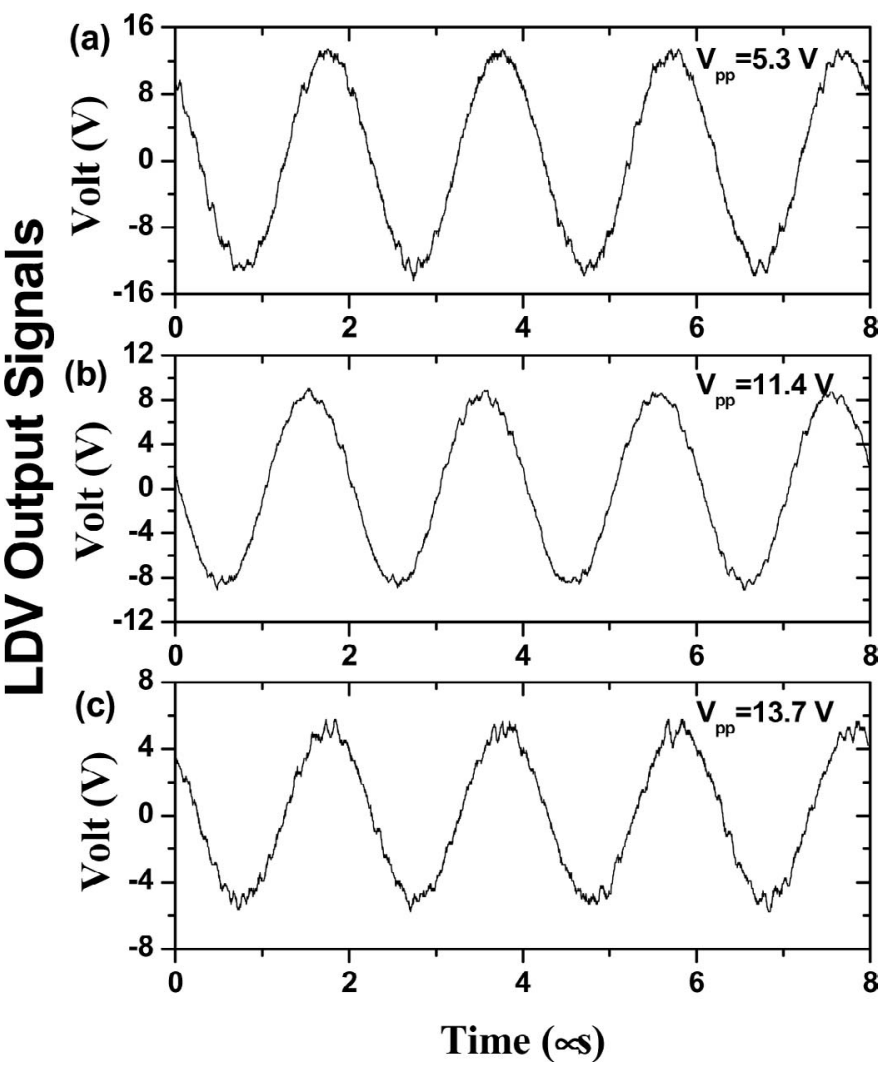

Fig. 7. Output signals of longitudinal vibration at the ultrasonic nozzle tip measured by LDV: (a) 5-Fourier horn, (b) 3-Fourier horn, and (c) 1-Fourier horn nozzles at respective peak-to-peak drive voltages of 5.3, 11.4, and $13.7 \mathrm{~V}$ and respective resonant frequencies of 505 , 498 , and $496 \mathrm{kHz}$. (Note: LDV output signal $1 \mathrm{~V}=0.25 \mu \mathrm{m}$ longitudinal displacement.)

and 1-horn nozzles, respectively. Groups 3 and 4 were identified as flexural modes.

To further verify the longitudinal mode of vibration, the vibration velocity at the nozzle tip along the nozzle axis (longitudinal vibration) was measured using the Polytec LDV at carefully tuned drive frequencies based on Table III. A strong, time-dependent voltage output as shown in Fig. 7(a) was detected only at the drive frequency of $505 \mathrm{kHz}$ for the 5 -horn nozzle. The voltage output was reduced to half when the drive frequency varied by $\pm 1 \mathrm{kHz}$. No signal was detected at other frequencies, including the resonant frequencies of 310 and $330 \mathrm{kHz}$ in which longitudinal vibration was predicted by the simulation. Thus, the longitudinal vibration measurement has unequivocally verified the simulation result of longitudinal vibration at the design frequency.

The output signal decreases as the electrode drive voltage decreases as shown in Fig. 8(a). Also shown in Fig. 8(a) are the measured output signals from the base of the 5horn nozzle. Dividing the output signal from the tip by that from the base yields an overall amplitude gain of 24 as shown in Fig. 8(a). The measured amplitude gain is in very good agreement with the respective predictions of 27 and $2^{5}$ by the simulation and the design. 


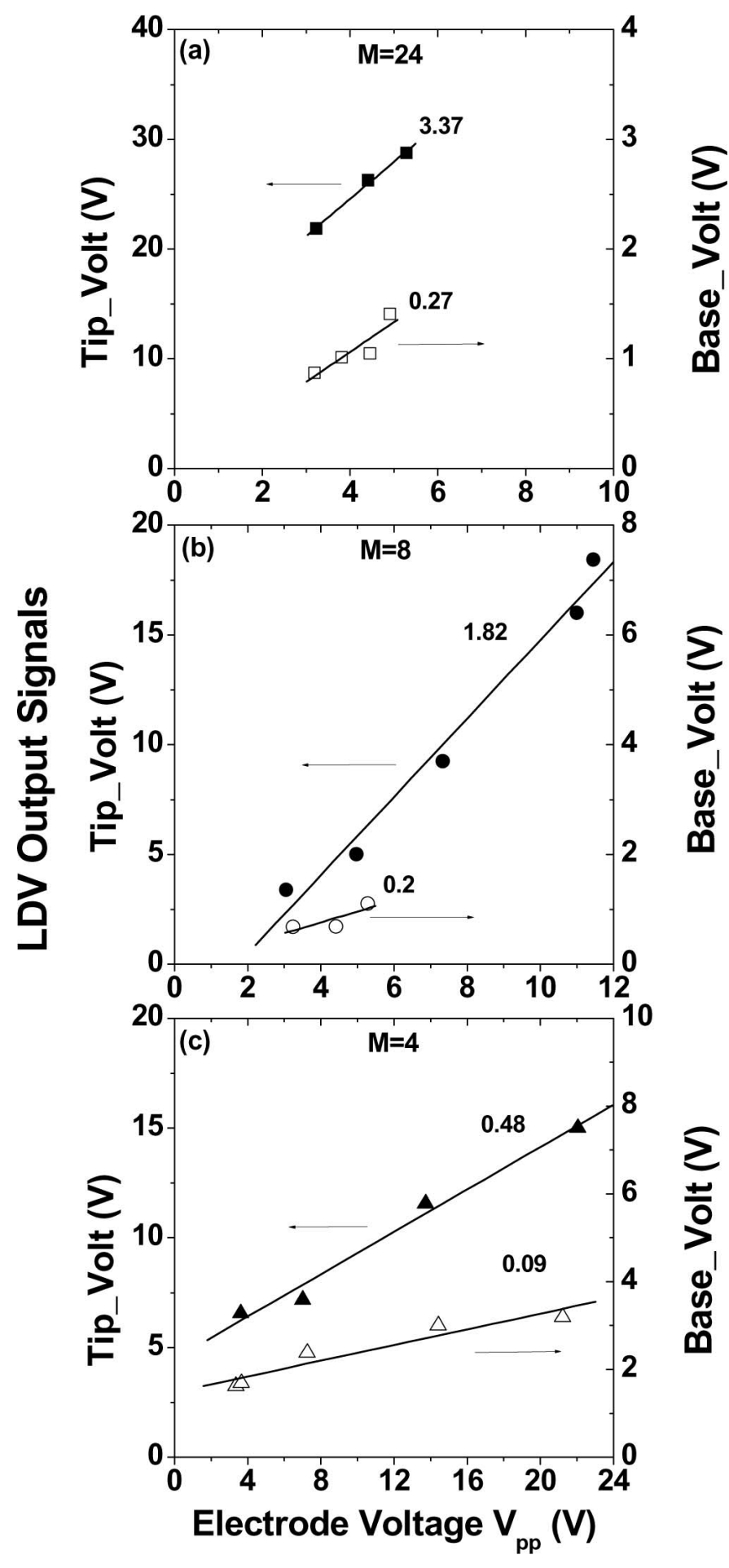

Fig. 8. Output signals of longitudinal vibration at the tips and bases of Fourier nozzles measured by LDV: (a) 5-Fourier horn, (b) 3-Fourier horn, and (c) 1-Fourier horn nozzles. Note that the numbers next to the lines are the slopes. (Note: LDV output signal $1 \mathrm{~V}=0.25 \mu \mathrm{m}$ longitudinal displacement.)
A comparison of Figs. 6(a) and (b) reveals that the frequency spectrum for the 5-horn nozzle is only slightly more complex than that of the 1-horn nozzle. The flexural modes of vibration at frequencies higher than the longitudinal mode in the 5-horn nozzle appear to shift to lower frequency than that in the 1-horn nozzle (582 versus $604 \mathrm{kHz}$ ). Nevertheless, because the frequencies of the neighboring flexural vibrations are far from those of the desired longitudinal vibration, no interference is expected. This expectation was indeed confirmed by the experimental results. Specifically, only a single frequency at $505 \mathrm{kHz}$ was obtained by the LDV measurement of longitudinal vibration from the tip of the 5-horn nozzle. Likewise, only one resonant frequency each was observed: $498 \mathrm{kHz}$ and $496 \mathrm{kHz}$ for the 3-horn and 1-horn nozzles, respectively. The corresponding time-dependent, LDV output signals are shown in Figs. 7(b) and (c). As in the 5-horn nozzle, the output voltage signal was reduced to half when the drive frequency varied by $\pm 1 \mathrm{kHz}$. No signal was detected at other frequencies, including group 1 frequencies in which longitudinal vibration was predicted by the simulation.

As shown in Figs. 7(a) and (b), the LDV output signal of the 5-horn nozzle saturates ( $30 \mathrm{~V}$ peak-to-peak equivalent to $7.5 \mu \mathrm{m}$ peak-to-peak displacement at $500 \mathrm{kHz}$ ) at a drive voltage of only $5.3 \mathrm{~V}$ peak-to-peak; and that of the 3-horn nozzle is only $18 \mathrm{~V}$ peak-to-peak (equivalent to $4.5 \mu \mathrm{m}$ peak-to-peak displacement) at a higher drive voltage (11.4 V peak-to-peak). The output signal of $18 \mathrm{~V}$ peak-to-peak from the 3-horn nozzle is also significantly higher than the $11 \mathrm{~V}$ peak-to-peak output signal (equivalent to $2.8 \mu \mathrm{m}$ peak-to-peak displacement) from the single horn nozzle at $13.7 \mathrm{~V}$ peak-to-peak drive voltage as shown in Fig. 7(c).

The output signals from the LDV measurements of the longitudinal vibrations of the 5-horn, 3-horn, and 1-horn nozzles at various drive voltages are shown in Figs. 8(a)(c), respectively. Solid and open data points are for measurements at the tip and the base of the nozzles, respectively. These figures show that all measurements are within the linear range of the instrument and, thus, the slope of each line also is shown in the figures next to the line. Fig. 8 shows that the measured amplitude magnifications of 24 and 8 for the 5-horn and 3-horn are in good agreement with the respective simulation results (27 and 8.5) and the design values $\left(2^{5}\right.$ and $\left.2^{3}\right)$. The measured amplitude gain for the 1-horn nozzle is unexpectedly high (4 versus 2 ), possibly due to experimental errors. Coupled with the small slope as shown in Fig. 8(c), the 1-horn nozzle is more likely to have transducer failure when large longitudinal vibration amplitude is required as in ultrasonic atomization. In contrast, the much larger slope as shown in Fig. 8(a) and the much larger amplitude magnification associated with the 5-horn nozzle provide its unique advantages for use in ultrasonic atomization.

In short, the detailed measurements of longitudinal vibration at the resonant frequency close to the design value have verified the simulation results that the amplitude gain 
at the nozzle tip increases as the number of Fourier horns $(\mathrm{n})$ in cascade increases. The findings are direct evidence of the resonance principle based on which the amplitude gain at the nozzle tip increases proportionally to $2^{\mathrm{n}}$.

\section{Conclusions}

The 3-D simulation has shown that silicon-based, megahertz, ultrasonic nozzles using multiple Fourier horns are capable of achieving much larger amplitude gain at the nozzle tip with no reduction in the tip cross-sectional area compared with single horn nozzles at the same resonant frequency. The vibration mode is longitudinal with insignificant lateral motion or bending. These theoretical predictions have been confirmed by both impedance analysis and longitudinal vibration measurement. Therefore, the required electric drive power and the risk of transducer failure of a 5-horn ultrasonic nozzle should be drastically reduced compared with a conventional megahertz ultrasonic nebulizer.

\section{ACKNOWLEDGMENT}

The authors would like to gratefully acknowledge Professor C. H. Yang at the Department of Mechanical Engineering, Chang Gung University, Taiwan, for use of the Polytec Laser Doppler Vibrometer.

\section{REFERENCES}

[1] A. Lal and R. M. White, "Micromachined silicon ultrasonic atomizer," in Proc. IEEE Ultrason. Symp., 1996, pp. 339-342.

[2] H. L. Berger, Ultrasonic Atomizing Nozzle Systems. Sono-Tek brochure, 1991.

[3] S. C. Tsai, P. Luu, P. Childs, and C. S. Tsai, "Ultrasoundmodulated twin-fluid atomization of a liquid jet," IEEE Trans. Ultrason., Ferroelect., Freq. Contr., vol. 46, pp. 139-146, 1999.

[4] S. C. Tsai, "Ultrasound-Modulated Two-Fluid (UMTF) Atomization," U.S. Patent \#5,687,905, 1997.

[5] S. C. Tsai, P. Luu, P. Childs, A. Teshome, and C. S. Tsai, "The role of capillary waves in two-fluid atomization," AIP Phys. Flu$i d s$, vol. 9, pp. 2909-2918, 1997.

[6] Y. L. Song, S. C. Tsai, C. Y. Chen, T. K. Tseng, C. S. Tsai, J. W. Chen, and Y. D. Yao, "Nanoparticles synthesis using air-assisted ultrasonic spray pyrolysis," submitted for publication.

[7] W. Eisenmenger, "Dynamic properties of the surface tension of water and aqueous solutions of surface active agents with standing capillary waves in the frequency range from $10 \mathrm{kc} / \mathrm{s}$ to $1.5 \mathrm{Mc} / \mathrm{s}$," Acustica, vol. 9, pp. 327-340, 1959.

[8] S. C. Tsai, P. Luu, P. Tam, G. Roski, and C. S. Tsai, "Flow visualization of Taylor-mode breakup of a viscous liquid jet," AIP Phys. Fluids, vol. 11, pp. 1331-1341, 1999.

[9] E. Eisner, "Design of sonic amplitude transformers for high magnification," J. Acoust. Soc. Amer., vol. 35, pp. 1367-1377, 1963.

[10] J. J. Wortman and R. A. Evans, "Young's modulus, shear modulus, and Poisson's ratio in silicon and germanium," J. Appl. Phys., vol. 36, pp. 153-156, 1965.

[11] B. A. Auld, "Piezoelectricity," in Acoustic Fields and Waves in Solids. vol. 1, New York: Wiley-Interscience, 1973.

[12] S. C. Tsai, T. K. Tseng, Y. F. Chou, H. Y. Tsai, C. S. Tsai, and J. H. Yoo, "High frequency silicon-based ultrasonic nozzle," in Proc. IEEE Ultrason. Symp., 2001, pp. 637-639.

[13] S. C. Tsai, T. K. Tseng, Y. L. Song, Y. F. Chou, C. S. Tsai, and P. Z. Chang, "Modeling and simulation of a high frequency
MEMS-fabricated ultrasonic nozzle," in Mater. Res. Soc. Symp. Proc. MEMS and Bio-MEMS, vol. 729, 2002, pp. 221-227.

Shirley C. Tsai received her B.S. degree in chemical engineering from the National Taiwan University, Taipei, in 1963 and her Ph.D. degree in physical chemistry from the California Institute of Technology, Pasadena, in 1969. She was with industrial research centers (Gulf R \& D Company in Pittsburgh, PA, and Occidental Research Center in Irvine, CA) for 10 years prior to joining California State University at Long Beach (CSULB) as a professor of chemical engineering in 1983. She has been a visiting professor at Academia Sinica, in Taipei, Taiwan since 1999.

Dr. Tsai has conducted basic research on atomization and rheology in the past 15 years through the sponsorships of National Science Foundation (NSF) and the Department of Energy. She has published one single-authored book on coal (by Elsevier Scientific Publisher, 1982) and some 80 research papers in the areas of ultrasoundmodulated, two-fluid (UMTF) atomization, high-frequency ultrasonic nozzles, air-assisted ultrasonic spray pyrolysis, Taylor-mode jet breakup, airblast atomization of coal water slurries, rheology of concentrated suspensions, two-phase flows, coal and mineral processing, radiation chemistry, and molecular spectroscopy. She holds 10 U.S. Patents on UMTF atomization, coal liquefaction, coal gasification, and shale oil recovery.

Her most recent research interests are micro electro mechanical system (MEMS)-based, high-frequency, ultrasonic nozzles to produce micron-sized sprays for applications to nanoparticles synthesis and spray drying of nano-, bio-dispersions. She is a member of the American Institute of Chemical Engineers, the Materials Research Society, the American Institute of Physics Society of Rheology, and the Institute of Liquid Atomization and Spray Systems. She received the 1987-1988 CSULB Distinguished Faculty Scholarly and Creative Activity Award and TRW Excellence in Teaching Award, and the CSULB Associated Engineering Students Body Outstanding Faculty Award in 1993 and 1997.

Yuan F. Chou received his Ph.D. degree in aeronautics and astronautics from Purdue University, Lafayette, IN, in 1980. He joined National Taiwan University, Taipei, as a visiting instructor of mechanical engineering in 1981 and was promoted to Professor in 1986.

His major research fields are vibration and acoustics with emphasis in micro electro mechanical system applications.

Chen S. Tsai (M'69-SM'77-F'83) is a naturalized citizen of the United States. He received his Ph.D. degree in electrical engineering from Stanford University, Stanford, CA, in 1965. He was with the Lockheed Palo Alto Research Labs, Palo Alto, CA, as a research scientist for $3 \frac{1}{2}$ years before joining Carnegie-Mellon University, Pittsburgh, PA, as an assistant professor in 1969. In 1974, he was promoted to Professor and was awarded an Endowed Chair Professorship in 1979. In 1980 he joined the University of California, Irvine, CA, (UC Irvine) as a Professor of electrical engineering, served as Acting Department Chair from 1985-1986, and was advanced to Professor, Above-Scale in 1991. During a 1999-2002 professional leave he served as the Distinguished Research Fellow and Founding Director of the Institute for Applied Science and Engineering Research with the Academia Sinica in Taipei, Taiwan.

Dr. Tsai has published some 370 research papers and 13 encyclopedia and book chapters. He was the editor and contributed three chapters to the first topical volume on Guided-Wave Acoustooptics, published by Springer-Verlag in 1990. Dr. Tsai's group at UC Irvine has conducted basic research on guided-wave electrooptics, acoustooptics and magnetooptics, acoustic microscopy, nano-scale iron film-based wideband integrated microwave devices, and Si-based high frequency atomizers and applications to nano particles synthesis and processing of bio nano dispersions through the sponsorships of the University of California Microelectronics Innovation and Computer Research Opportunities (UC MICRO)/Discovery/Industry 
programs, the National Science Foundation (NSF), the National Security Agency, the Army Research Office, the Air Force Office of Scientific Research, and the Office of Naval Research.

Among the awards and honors he has received are the Fellows of the IEEE, the Optical Society of America, the American Association for Advancement of Science (AAAS), and the Society of Photo-Instrumentation Ingineers (SPIE), the IEEE Distinguished
Lectureship Award, IEEE Society Best Paper Award, UC Irvine Faculty Senate Distinguished Research Lectureship Award, UC Irvine Lauds/Laurels Research Award, and the International Microoptics Award. He is an academician of the Academia Sinica in Taiwan, a foreign member of the Russian Academy of Engineering Sciences, and a foreign honorary member of the Russian Popov Society. 